US Army Corps

of Engineers ${ }_{\circledast}$

\title{
A Robust, Scalable Framework for Conducting Climate Change Susceptibility Analyses
}

by Eric R. Britzke, Nathan R. Beane, Austin V. Davis, Michael D. Whitby, and Sandra M. Brasfield

PURPOSE: Resource managers must consider climate change during their planning processes as future landscapes have the potential to vary greatly from current conditions. Military training and testing lands are extremely important and must be assessed for potential impacts of climate change. Climate change susceptibility analyses are commonly limited in their scope, thereby requiring multiple independent analyses within the same region. Without a standardized, comprehensive analysis method, the ability to prioritize areas susceptible to climate change within a region of interest is hindered. This deficit limits the applicability of results as well, further illustrating the need to develop a standardized method that incorporates multiple factors into a scalable framework to provide a more complete climate change susceptibility analysis. This framework must allow individual users the option of weighting environmental variables differently based on conservation concerns in managed areas. In this technical note, the authors present a method that allows multiple stakeholders - each with their own management requirements and concerns for assessing susceptibility - to use a common framework to assess climate change susceptibility. This framework enables improved analytical results of climate change to be incorporated into long-term regional conservation planning. The framework should prove to be a valuable tool for resource managers faced with integrating the potential impacts of climate change into their natural resource plans.

INTRODUCTION: Potential impacts of climate change are receiving a great deal of attention as resource managers attempt to plan for predicted changes (e.g., Spittlehouse and Stewart 2003, Millar et al. 2007, Rustad et al. 2012). While substantial changes may take decades to appear, more rapid changes to both plant and animal ranges have already been extensively documented (e.g., McKenney-Easterling et al. 2000, Walther et al. 2002, Kelly and Goulden 2008, Woodall et al. 2009, Chen et al. 2011). Research has shown varying degrees of change across the landscape, including changes in flowering phenology (Bradley et al. 1999), temperature regimes (Barber et al. 2000), and hydrologic cycles (IPCC 2007, Knapp et al. 2008, Walker et al. 2011). As these environmental and ecosystem impacts take place - as a result of changing climatic conditions the tools for identifying areas of heightened risk from varying forms of climate forcings is needed.

Based on global climate model projections, deviations from current climate conditions are expected to intensify over the next century (IPCC-AR4 2007). Additional impacts from climate change are likely to place further restrictions on land management activities and lead to increased susceptibility to invasive plants, exotic invasive forest pests, and diseases (Rosenzweig et al. 2001; Hellman et al. 2008). Even though observed changes may not be evident in some localities for several decades, land managers should be aware of potential changes to the landscapes so that 
appropriate mitigation and management strategies to minimize future impacts can be implemented.

Climate change susceptibility analyses are typically addressed in two ways: 1) efforts focused primarily on the extent of climatic change (e.g., change in temperature and precipitation, storm frequency, sea-level rise); and 2) efforts focused on the loss of unique habitats or targeted species of conservation concern within a given ecosystem. While these factors are influenced by the changes in climate, some are influenced by changes in distant parts of the world (e.g., sea level rise resulting from warming in the arctic), or may be near a tipping point such that a small change may result in a disproportionate shift in the impact (Chen et al. 2011; Joshi et al. 2012). Thus, climate change analyses that focus solely on temperature and precipitation changes and ignore other real impacts may not fully represent the true vulnerability of a region to climate change. Additionally, focusing on a single species or habitat fails to provide a holistic assessment of impacts to a region.

Consequently, a GIS-based methodology was developed that is capable of incorporating various data sources indicating potential impacts of climate change (either directly or indirectly) into a single cohesive susceptibility analysis. Specifically, this methodology allows the user to assess data sets collected in different units to project future conditions at varying spatial resolutions. This technical note provides a framework to integrate multiple data sources while still allowing the user to acquire only necessary data sources pertinent to the aspect of climate change $\mathrm{s} / \mathrm{he}$ is examining (e.g., predicted precipitation change weighted higher than predicted temperature change for flood risk assessments). This option allows the user to customize the risk vulnerability score based on variable importance, thereby highlighting which factors pose the greatest risk under predicted future environmental conditions. This technique has allowed the creation of a susceptibility analysis method that can be used by interested groups with different priorities, while still enabling users to assess their results in the geographic context of a larger effort.

METHODOLOGY: There are multiple steps to developing climate change susceptibility analysis using this framework. Initial steps involve generating a list of factors that will be incorporated into the analysis. The user should first determine the spatial extent in which the analyses will be conducted, with a larger scale recommended, as climate data is broadly generalized at localized levels. Next, users should acquire the necessary data (Figure 1, Step 1). This may involve using publically available data (e.g., climate data from www.climatewizard.com) or incorporating specific data layers (e.g., vegetation changes, sea level rise, and flood risk) used in previous susceptibility analyses and potentially at different scales of resolution. In some circumstances, this process may identify data needs that must be met for a complete susceptibility analysis to be conducted.

Next, users need to obtain predicted data for a future time period of interest. Climate projections are typically available as 30-year average estimates, with the year 2080 often reported for endof-century estimates; the timespan typically runs from 2070-2099. These future projections allow the user to calculate a percentage change from current conditions to a future time scale of interest (Figure 1, Step 2). Once completed across the study area, the user normalizes values by dividing all percent changes by the maximum value for that parameter. This puts all of the parameters on the same scale for later analyses. Once the normalization process is completed for each of the parameters, the parameters should be included into a single susceptibility analysis by 


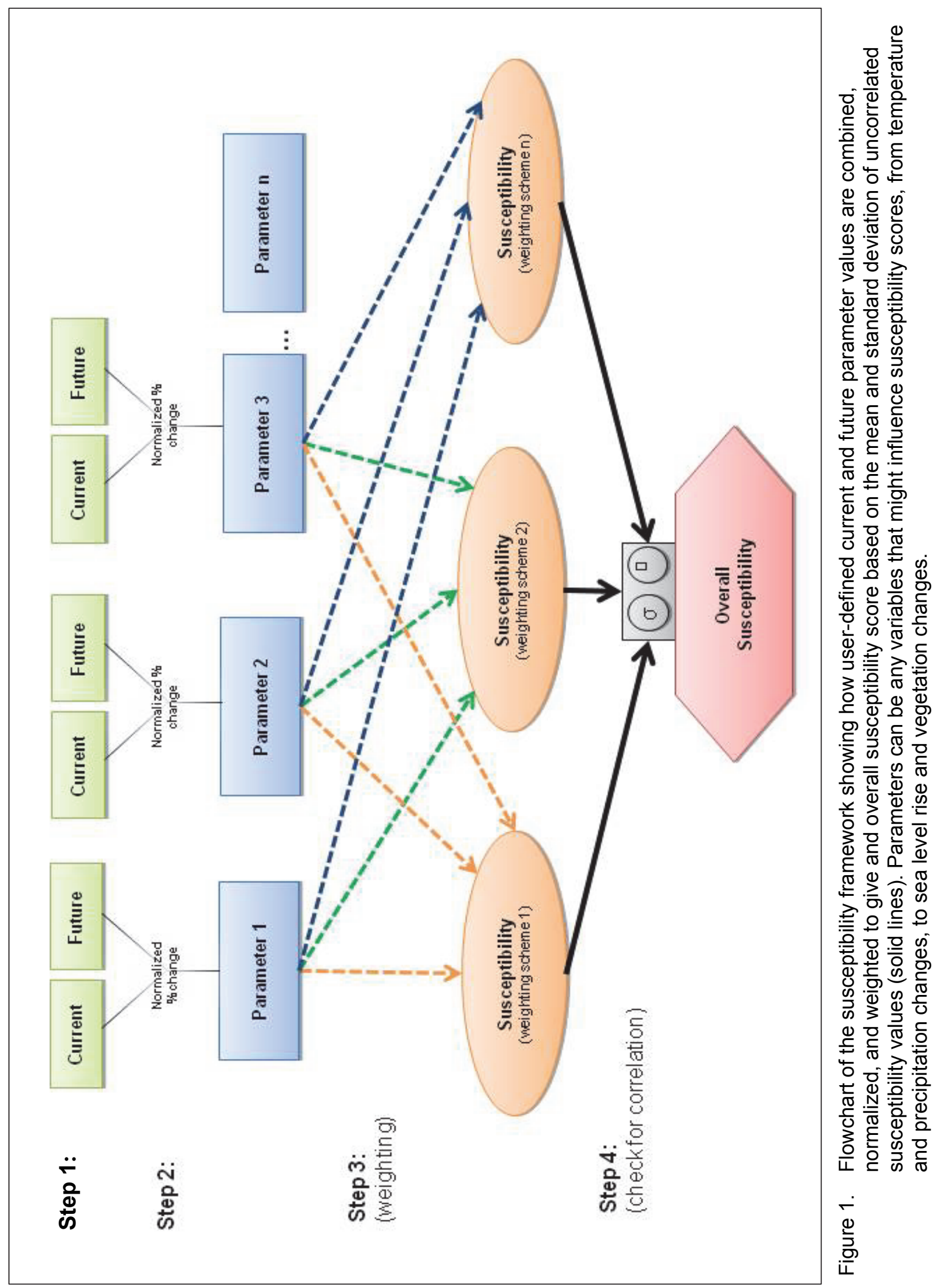


establishing a series of weighted coefficients (Figure 1, Step 3). These weighted coefficient values can be as simple or as complex as desired, but each set of parameters should adhere to the same weighting scenario. Therefore, when combined vulnerability scores are calculated, the user will have a more robust measure of combined susceptibility. For example, in a three-parameter situation, if one weighting assignment was $80 \%, 10 \%, 10 \%$, then each of these weighting scenarios should be used on each parameter $(80 \%$ used for each parameter, three total sets of weightings). Using the weighting schemes developed, the susceptibility scores for a grid cell are calculated by the following formula:

\section{Equation 1:}

Susceptibility Value $=W_{1} V 1+W_{2} V 2+W_{3} V 3 \ldots \ldots W n V n$
where,
$W_{1}=$ Parameter \#1 Weighting
$V_{1}=$ Parameter \#1 Value
$W_{2}=$ Parameter \# 2 Weighting
$V_{2}=$ Parameter \#2 Value
$W_{3}=$ Parameter \#3Weighting
$V_{3}=$ Parameters \#3Value
$W_{n}=$ Parameter $n$ Weighting
$V_{n}=$ Parameters $n$ Value

As raster-based grid cells are generally utilized in climate vulnerability analyses for a variety of reasons, primarily due to processing advantages, the authors use raster-based grid size as the computational area. The grid size incorporated for an analysis can be variable in size due to multiple scales of data analyzed; however, the user should be cautioned that the resolution of the data is only as good as the largest grid cell size used and that resampling data to this extent is recommended. The calculation of the susceptibility scores for each grid cell and for each weighting scenario examined results in a large number of susceptibility scores calculated. The user can go through a data reduction effort to focus on scores that are independent of one another. This removes weighting schemes that are not statistically different from one another, leaving only weighting scenarios that provide independent and meaningful results in the overall susceptibility analyses (Figure 1, step 4). One approach for this data reduction technique is to conduct pairwise correlation analyses. For sets of data where the weighting scenario for all parameters is shown to be highly correlated with another weighting scenario, one of the weighting scenarios is removed. For example, if there were three parameters being used, and the result of a $70 \%, 15 \%, 15 \%$ weighting scenario is similar to another weighting scenario, one of these is arbitrarily chosen to be removed. These comparisons are made until all of the weighting scenarios that remain are statistically different, maximizing the range of values obtained while minimizing the number of weighting scenarios used. Completing the weighting scenario reduction process programmatically allows the user to derive actionable information as efficiently as possible. Finally, an average and variation of susceptibility metrics can be calculated to evaluate and compare regions of interest (Figure 1). 
DISCUSSION: This assessment technique provides a framework that can be used by natural resource managers and scientists to analyze the effects of climate change with respect to ecosystem vulnerabilities. The vulnerabilities can be caused by direct changes in climate or changes in other specific factors, such as sea level rise, vegetation changes, and flood risk. Further, this method allows for weighting scenarios to be applied to multiple parameters of interest to assess an array of ecosystem vulnerabilities and identify areas with highly variable or sensitive responses to climate change.

An important part of this process is the normalization of values of each parameter examined. Since most of these parameters are reported initially as a percentage change from the current condition, there is a need to equalize the potential effects of any given factor. Although the percent change may be different among parameters, the impact of that change is unknown; thus, the largest percentage change may not have the largest impact. Through the process of normalization, the parameters can be equally weighted for comparative analyses across multiple scales and variables incorporated, allowing the user the flexibility to assess how climate susceptibility is affected by variable weightings of select parameters.

While this framework provides an opportunity to easily combine multiple data sources — that are often freely available from many federal, state, and global agencies - into a single climate change susceptibility analysis, the scalable methodology allows for updates to the initial analysis as new data are obtained, creating a robust framework for assessing climate vulnerabilities by incorporating both external and internal forces on ecosystems. Further, the user can alter the weighting coefficient of a select variable to determine where the greatest impact on the landscape is likely to occur for that variable in the context of an overall susceptibility analysis, where all considered variables are weighted equally. Having an understanding of the average susceptibility scores as well as the variation in the scores across the scenarios can give natural resource managers the ability to prioritize areas with a high chance of success regardless of the specific scenario utilized.

SUMMARY: Using the approach described herein, it is possible for land managers and decision makers to use GIS-derived data to assess overall susceptibility for an area of interest and provide an overlay analysis to determine regional susceptibility to climate change within varying land management frameworks. This analytical method provides a framework that incorporates multiple data sources into a unique climate change susceptibility analysis framework that can be conducted on a regional scale and be readily adapted as new data becomes available. Thus, a single metric or combination of metrics can be calculated and dynamically visualized. Synthesizing data from a variety of sources, this GIS framework provides the user with a powerful tool for projecting climate change impacts.

POINTS OF CONTACT: For additional information, contact: Dr. Eric R. Britzke, (601) 6343641,Eric.R.Britzke@usace.army.mil.This technical note should be cited as follows:

Britzke, E.R., N.R. Beane, A.V. Davis, M.W. Whitby, and S.M. Brasfield. A robust scalable framework for conducting climate change susceptibility analyses. ERDC TN-14-1. Vicksburg, MS: US Army Engineer Research and Development Center. 
ACKNOWLEDGEMENTS: Two anonymous reviewers provided valuable comments to the manuscript, making quality improvement to the final document. The work was supported by the U.S. Army's Environmental Quality Technology Applied and Advanced Research Program (Dr. Elizabeth Ferguson, Technical Director).

\section{REFERENCES}

Barber, V.A., G.P. Juday, and B.P. Finney. 2000. Reduced growth of Alaskan white spruce in the twentieth century from temperature-induced drought stress. Nature 405:658-673.

Bradley, N.L., A.C. Leopold, J. Ross, and W. Huffaker. 1999. Phenological changes reflect Climate change in Wisconsin. Proc. Natl. Acad. Sci. 96:9701-9704.

Chen, I-C., J.K. Hill, R. Ohlemuller, D.B. Roy, and C.D. Thomas. 2011. Rapid range shifts of species associated with high levels of climate warming. Science 333(6045):1024-1026.

Hellmann, J. J., J.E. Byers, B.G. Bierwagen, and J.S. Dukes. 2008. Five Potential Consequences of Climate Change for Invasive Species. Conservation Biology 22: 534-543.

IPCC. 2007. Climate change 2007: the physical science basis. In: Solomon, S., Quin, D., Manning, M., Chen, Z., Marquis, M., Avery, B., Tignor, M., Miller, H.L. (eds.), Contribution of working group I to the fourth assessment report of the Intergovernmental Panel on Climate Change. Cambridge, United Kingdom and New York, USA: Cambridge University Press.

IPCC-AR4. 2007. Climate change 2007: synthesis report. Contribution of working groups I, II, and III to the fourth assessment report of the Intergovernmental Panel on Climate Change. Geneva, Switzerland.

Joshi, P. K., A. Rawat, S. Narula, and V. Sinha. 2012. Assessing impact of climate change on forest cover type shifts in Western Himalayan Eco-region. Journal of Forestry Research 23:75-80.

Kelly, A.E., and M.L. Goulden. 2008. Rapid shifts in plant distribution with recent climate change. Proceedings of the National Academy of Sciences [PNAS] 105(33):11823-11826.

Knapp, A.K., C. Beier, D.D. Briske, A.T. Classen, Y. Luo, M. Reichstein, M.D. Smith, S.D. Smith, J.E. Bell, P.A. Fay, J.L. Heisler, S.W. Leavitt, R. Sherry, B. Smith, and E. Weng. 2008. Consequences of more extreme precipitation regimes for terrestrial ecosystems. Bioscience 58(9):811-821.

McKenney-Easterling, M., D.R. Dewalle, L.R. Iverson, A.M. Prasad, and A.R. Buda. 2000. The potential impacts of climate change and variability on forests and forestry in the Mid-Atlantic Region. Climate Research 14:195-206.

Millar, C. I., N. L. Stephenson, and S. L. Stephens. 2007. Climate change and forests of the future: managing in the face of uncertainty. Ecological Applications 17(8): 2145-2151.

Rosenzweig, C., A. Iglesias, X. B. Yang, P. R. Epstein, and E. Chivian. 2001. Climate change and extreme weather events: implications for food production, plant diseases, and pests. Global Change and Human Health 2:90-104. 
Rustad, L., J. Campbell, J.S. Dukes, T. Huntington, K.F. Lambert, J. Mohan, and N. Rodenhouse. 2012. Changing climate, changing forests: the impacts of climate change on forests of the northeastern United States and eastern Canada. Gen. Tech. Rep. NRS-99. Newtown Square, PA: U.S. Department of Agriculture, Forest Service, Northern Research Station.

Spittlehouse, D. L. and R. B. Stewart. 2003. Adaptation to climate change in forest management. $B C$ Journal of Ecosystems and Management 4(1): 1-11.

Walker, J.F., Hay, L.E., and S.L. Markstrom. 2011. Characterizing climate-change impacts on the 1.5-yr flood flow in selected basins across the United States: a probabilistic approach. Earth Interactions 15, Paper 18.

Walther, G.-R., E. Post, P. Convey, A. Menzel, C. Parmesan, T.J.C. Beebe, J.-M. Fromentin, O. HoeghGuldberg, and F. Bairlein. 2002. Ecological responses to recent climate change. Nature 416:389-395.

Woodall, C.W., C.M. Oswalt, J.A. Westfall, C.H. Perry, M.D. Nelson, and A.O. Finley. 2009. An indicator of tree migration in forests of the eastern United States. Forest Ecology and Management 257:1434-1444.

NOTE: The contents of this technical note are not to be used for advertising, publication, or promotional purposes. Citation of trade names does not constitute an official endorsement or approval of the use of such products. 